Rhode Island College

Digital Commons @ RIC

\title{
Perceived Barriers to End-of-Life Care Discussions in Cancer Patients from the Perspective of Ambulatory Oncology Nurses
}

Susan K. Hobin

Rhode Island College

Follow this and additional works at: https://digitalcommons.ric.edu/etd

Part of the Other Nursing Commons

\section{Recommended Citation}

Hobin, Susan K., "Perceived Barriers to End-of-Life Care Discussions in Cancer Patients from the Perspective of Ambulatory Oncology Nurses" (2017). Master's Theses, Dissertations, Graduate Research and Major Papers Overview. 182.

https://digitalcommons.ric.edu/etd/182

This Major Paper is brought to you for free and open access by the Master's Theses, Dissertations, Graduate Research and Major Papers at Digital Commons @ RIC. It has been accepted for inclusion in Master's Theses, Dissertations, Graduate Research and Major Papers Overview by an authorized administrator of Digital Commons @ RIC. For more information, please contact digitalcommons@ric.edu. 


\section{PERCEIVED BARRIERS TO END-OF-LIFE CARE DISCUSSIONS IN CANCER PATIENTS FROM THE PERSPECTIVE OF AMBULATORY ONCOLOGY NURSES}

by

Susan K. Hobin

A Major Paper Submitted in Partial Fulfillment

of the Requirements for the Degree of

Master of Science in Nursing

in

The School of Nursing

Rhode Island College

2017 


\begin{abstract}
With the advancing age of the population, the increasing incidence of cancer, and all of the advances in cancer therapy, comes the ability to prolong life in cancer patients well beyond what was possible even ten years ago. Along with the progress of advanced surgical and radiation techniques, there is now an ever-expanding array of chemotherapy, biotherapy, and immunotherapy being used on patients in all age groups and in all stages of cancer. These advances have thrilled researchers and physicians, as they now have a multitude of options to offer cancer patients, perhaps even as late as third or fourth line therapy. If traditional chemotherapy fails, it is very likely that a new novel agent will be offered to the patient, regardless of age and performance status. Cancer patients often have an unwavering amount of hope and faith in their physician, and these treatments, while promising and encouraging, add to the growing problem of delaying end-of-life care discussions in patients, often until they are exhausted of all hope and quality of life. The Institute of Medicine has made dying well from cancer a top priority and states that those with palliative needs deserve access to expert-level care and those who meet the criteria of a six month or less prognosis should be offered hospice. Ambulatory oncology nurses are in a unique position to add to the discussion of barriers to end-of-life care discussions in cancer patients and, for this reason a qualitative research study was conducted.
\end{abstract}




\section{Acknowledgements}

First, I would like to thank Linda Dame for her help, guidance, and support in helping me to write this paper. Linda, you have been instrumental in getting me through this process and I so appreciate your promptness and your feedback. Your kindness and encouragement throughout this process was invaluable to me and I am grateful to have had you as my first reader.

I would also like to thank my husband, Michael, for the unconditional support and encouragement he has given me over the past four years. Mike, you are the reason I applied to the graduate program in the first place and I will be forever grateful to you for always believing in me and for putting up with me, even when I didn't deserve it. You are my role model for hard work and perseverance, and you inspire me daily to try to be the best version of myself. As you always say, "In order to finish you must begin". Well, I did it, and I am so grateful to you for always believing in me. Thank you for being my rock and my best friend in the world. I love you more than you could ever know, and by the way, I think it may finally be time to replace the red chair! 


\section{Table of Contents}

Background/Statement of the Problem ...................................................................... 1

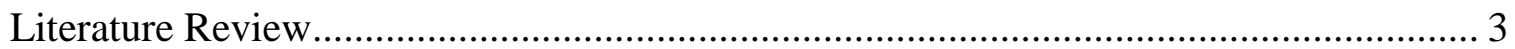

Theoretical Framework .................................................................................... 17

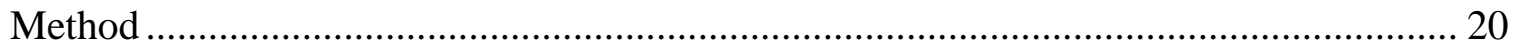

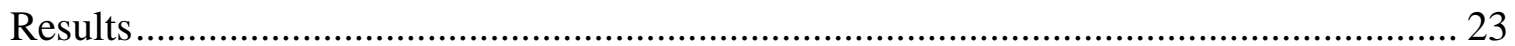

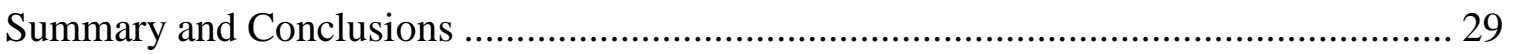

Recommendations and Implications for Advanced Nursing Practice ............................ 33

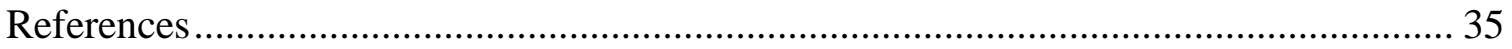

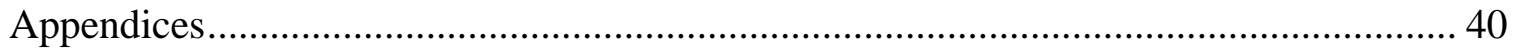


Perceived Barriers to End-of-Life Care Discussions in Cancer Patients from the Perspective of Ambulatory Oncology Nurses

\section{Background/Statement of the Problem}

In 2016, an estimated 1,685,210 new cases of cancer will be diagnosed in the United States (U.S.) and 595,690 people will die from the disease (National Cancer Institute (NCI), 2016). Cancer is among the leading causes of death worldwide, is the second leading cause of death in U.S., and is the leading cause of death in those between the ages of 45 and 64 years (Kochanek, Jiaquan, Murphy, Minino, \& Hsiang-Ching, 2011). Dying well from cancer in America is a top priority for the Institute of Medicine (IOM). A few items from the IOM Committee's Proposed Core Components of Quality End-of-Life Care (2014) state that those with palliative needs deserve access to appropriate expert-level care and those who meet the criteria of a six-month or less prognosis should be offered hospice referral (IOM, 2014). As a country, the U.S. is falling short of the IOM's goals. Hospice care is underused in the U.S. and patients who are referred to hospice are often referred too late (Cohen \& Nirenberg, 2011). In addition, many oncologists tend to refer patients to specialized palliative care services only for uncontrolled symptoms or late in the course of disease when planning for hospital discharge (Greer, Jackson, Meier, \& Temel, 2013).

Barriers to implementation of early end-of-life (EOL) care in oncology have been reviewed extensively in the literature. In a study of 300 advanced practice oncology nurses on their knowledge, attitudes, and practice behaviors of advance care planning, nurses cited barriers such as: patients' themselves, families dynamics, and physicians' reluctance to have EOL discussions as reasons for EOL goals not being met with patients (Zhou, Stoltzfus, Houldin, Parks, \& Swan, 2010). Ambulatory oncology nurses are in a 
unique position to add to the discussion of barriers in EOL care due to the extensive amount of time spent with cancer patients during chemotherapy infusions. In a study by Davis, Lind, \& Sorenson (2013), which examined burnout among oncology nurses, there was a high level of emotional exhaustion found among nurses who worked in an outpatient setting. The emotional exhaustion may be due to the fact that the nurses develop longer and more personal relationships with their patients, resulting in feelings of greater anguish and helplessness compared to nurses who work in inpatient settings (Davis, Lind, \& Sorenson, 2013).

Although there is much in the literature about oncology nurses and their perceptions of EOL care, there is a gap in the literature regarding barriers to EOL care discussions from the perspective of ambulatory oncology nurses, who are often witnesses to patient's deterioration as the EOL approaches. Ambulatory nurses may not have opportunities to participate in discussions about EOL. This may be due to a lack of privacy in their setting, or a lack of accessibility to clinicians in an outpatient setting. Therefore, the purpose of the study was to explore barriers to end-of-life care discussions in cancer patients from the perspective of ambulatory oncology nurses. 


\section{Literature Review}

A literature review was conducted using the databases CINAHL, PubMed, and Medline. Search terms used were: oncology nurses, ambulatory oncology nurses, outpatient oncology nurses, barriers, obstacles, experiences, attitudes, perceptions, end-oflife, conversations, discussions, communications, palliative and hospice care. Various combinations of the search terms were utilized. Current literature was searched between 2006 and 2016 in order to explore barriers to end-of-life discussions in cancer patients. Subheadings addressed in the literature review were: Cancer, Hospice and Palliative Care, Utilization of Earlier Palliative and Hospice Care, End-of-Life Discussions in Cancer Patients, and Oncology Nurses' Perceptions of EOL Discussions.

\section{Cancer}

Cancer is a group of diseases characterized by the uncontrolled growth and spread of abnormal cells. If the spread is not controlled, it can result in death (American Cancer Society [ACS], 2016a). Cancer is among the leading causes of death worldwide. Cancer mortality is higher among men than women, and approximately 39.6 percent of men and women will be diagnosed with cancer at some point during their lifetimes (NCI, 2016). Cancer is caused by external factors such as tobacco, infectious organisms, an unhealthy diet, as well as inherited genetic mutations, hormones, and immune conditions (ACS, 2014). National expenditures for cancer care in the U.S. totaled nearly $\$ 125$ billion in 2010 and are projected to reach $\$ 156$ billion in 2020 (NCI, 2016).

\section{Hospice and Palliative Care}

Within the U.S., hospice care is available for those with an anticipated life expectancy of six months or less (ACS, 2016b). Once certified by a physician, hospice 
care can be provided in a variety of settings to include inpatient, home, extended care facility, or freestanding hospice facilities. Hospice care is designed to support individuals in the final stage of life when aggressive or curative treatments are no longer appropriate and when the focus shifts to quality of life and relief from physical and psychological pain (ACS, 2016b). In the U.S., the word "hospice" is interchangeable or synonymous with EOL, and some patients will make the transition from curative-based care to hospice care during their lifespan (Moir, Roberts, Martz, Perry, \& Tivis, 2015).

Palliative care is care for adults and children with serious illness that focuses on relieving suffering and improving quality of life for patients and their families, but is not intended to cure the disease itself. It provides patients of any age or disease stage with relief from symptoms, pain, and stress, and should be provided along with curative treatment (ACS, 2014). Palliative care can be provided within hospitals, extended-care facilities, clinics, and home care settings. It may be appropriate for anyone with a chronic progressive disease when symptom management becomes a challenge (Bonebrake, Culver, Call, \& Ward-Smith, 2010). Delineating between palliative care and hospice care is often difficult, which is a result of the overlap between the two and the proven reluctance of healthcare professionals to initiate palliative care or hospice referrals to patients with life-limiting illnesses (Bonebrake et al., 2010).

\section{Utilization of Earlier Palliative and Hospice Care}

While advances in cancer therapy hold promise for arresting disease progression, the state of science and research development has lagged in targeting the physical, psychosocial, and existential elements of living longer with advanced cancer (Greer et al., 2013). Emerging evidence has shown that the introduction of palliative care services early 
in the course of advanced cancer improves a number of outcomes, such as quality of life and mood, and decreases symptom burden and use of health care services (Greer et al., 2013). As a result, an expert panel of the American Society of Clinical Oncology (ASCO) recently recommended that patients with metastatic non-small cell lung cancer (NSCLC) should be offered concurrent palliative care along with standard oncologic care and that such combined treatment should be considered for any patients with metastatic cancer early in the course of the disease (Smith et al., 2012). Seven published randomized trials demonstrate the feasibility of providing various components of palliative care alongside usual oncology care, all showing evidence of benefit to patients with cancer. Such benefits include: equal to improved survival, reduced depression, improved caregiver and/or patient quality of life (QOL), and overall lower resource use and cost because EOL hospitalizations were avoided (Smith et al., 2012).

Von Gunten, Lutz, \& Ferris (2011) conducted a systematic review. The review consisted of 15 articles which were a mix of qualitative and quantitative studies. The purpose of the article was to examine the QOL conundrum that exists in the U.S. Their findings concluded that although national guidelines have consistently suggested that referral for hospice care is the best approach for medical management of patients with EOL needs, only about half of all cancer patients who die are ever referred for such care. Additionally, patients who are referred often live for only a few days, thereby undermining the potential benefits to patients and their families of a concerted approach to care by the hospice team over a lengthier period of time (Von Gunten et al., 2011). The abundance and complexity of newer chemotherapy regimens has also contributed to delays in earlier palliative and hospice care use. Even when patients clinical status has begun to wane, they 
will often pursue salvage regimens with the hope of response, and the path of least resistance for some oncologists may involve delivery of chemotherapy as a type of "placebo" rather than engage in a lengthy, honest, and emotionally draining discussion about prognosis with the patient and family (Von Gunten et al., 2011).

Sharma, Sharma, Wojtowycz, Wang, \& Gajra (2015) conducted a retrospective chart review on the utilization of palliative care and acute care services in older adults with advanced cancer. They studied 567 veterans who died with advanced cancer between 2002 and 2009 at the Syracuse Veterans Affairs Medical Center in New York. The authors concluded that barriers to accessing earlier palliative and hospice care included the lack of education of physicians and other health care providers regarding EOL and its management, providers personal and religious beliefs, as well as patient/family misinformation and aspects of the hospice system that limit the number and types of patients deemed appropriate for EOL care (Sharma et al., 2015). In the same study, researchers also found that there continues to be significant utilization of acute care services in all patients with advanced cancer and that the education of oncology providers in discussing goals of care and the integration of earlier palliative and hospice care services into our health care system is imperative (Sharma et al., 2015).

\section{End-of-Life Discussions in Cancer Patients}

The Study to Understand Prognoses and Preferences for Outcomes and Risks of Treatment (SUPPORT) trial was one of the first studies to examine EOL in hospitalized patients and identified EOL care obstacles leading to a decreased QOL for terminally ill patients (Connors et al., 1995). Among these obstacles was faulty communication. This was identified as a major shortcoming in the delivery of quality EOL care to patients. 
National guidelines recommend that physicians discuss EOL care planning with patients who have incurable cancer and a life expectancy of less than one year (National Comprehensive Cancer Network [NCCN], 2011). Despite the guidelines, a major study done by The Cancer Outcomes Research and Surveillance Consortium (CanCORS) found that physicians initiated EOL discussions a median of 33 days before death (Mack et al., 2012). CanCORS studied 1,231 patients with stage IV lung or colorectal cancer who survived at least 1 month after diagnosis. Patients in the study were identified as having had an EOL care discussion if a discussion about hospice or resuscitation was reported in the baseline or follow-up interview. Of the patients studied, $88 \%$ had EOL care discussions documented in medical records or reported by patients or surrogates, including $87 \%$ of patients who died during the study period. In contrast to national guidelines, however, conversations took place a median of about one month before death (Mack et al., 2012). Findings of the CanCORS study also concluded that acute medical deterioration, and not the diagnosis of incurable cancer, triggers physicians to discuss EOL care with patients. In addition, individuals who had EOL discussions with their physicians before the last 30 days of life were less likely to receive aggressive treatment, including chemotherapy, in their final days (Mack et al., 2012). Results of the study concluded that future efforts should focus on ways to initiate EOL conversations earlier in the disease trajectory (Mack et al., 2012). Limitations of the CanCORS study were that the depth or quality of discussions were not evaluated and that only the EOL topics of hospice and resuscitation were included as topics of discussion.

Keating et al. (2010) conducted a national survey of physicians caring for cancer patients. Items surveyed included timing of discussions regarding prognosis, Do Not 
Resuscitate (DNR) status, hospice, and preferred site of death with their terminally ill patients. The study had 4,074 respondents and was part of a national multi-regional study conducted by the CanCORS. CanCORS examined processes and outcomes of care for a population based cohort of cancer patients that included more than 10,000 patients diagnosed with lung or colorectal cancer during 2003-2005. Keating et al., (2010) noted that almost half of the physicians reported they would not discuss EOL options with terminally ill patients who are feeling well. Instead they would wait for symptoms to appear or until there are no more treatments left to offer (Keating et al., 2010). Although studies have shown that EOL discussions do not harm patients and even have positive outcomes for patients, some physicians who are aware of national guidelines delay EOL discussions because they fear that discussing death early in a patient's terminal course may be associated with less hope and poorer outcomes (Keating et al., 2010). Other findings of the study include that a significant minority of physicians reported that they would discuss prognosis or preferred site of death only if the patient or family initiated the conversation. Younger physicians, as well as physicians who did not specialize in oncology, reported discussing EOL options sooner than older ones and those who were oncology specialists (Keating et al., 2010).

Laryionava, Heubner, Hiddemann, \& Winkler (2014) surveyed a group of 29 oncology physician providers in Germany and found that health-care professionals differed considerably in their understanding of when to initiate discussions about forgoing cancer therapy at the EOL. Interviews were open-ended, and results showed that despite agreement that EOL discussions should occur earlier in the disease trajectory, there was little consensus as to what "earlier" should be. Responses ranged from "at the beginning of 
systemic therapy" to "when all therapy options have been exhausted" (Laryionava et al., 2014). Physicians worried that early discussions about forgoing cancer-specific therapy could have a negative impact on the patient-physician relationship, and some felt that, over time, the patient and physician could come to a "mutual agreement" on forgoing therapy without addressing the topic explicitly. Oncologists in the study suggested that they would initially mention situations in which forgoing treatment would be sensible, such as when the patient still feels well, and readdress the point again when only one treatment option is left or when the patient's condition worsens (Laryionava et al., 2014).

A review of the literature was conducted by Trice \& Prigerson (2009) regarding communication in end-stage cancer. Over 70 studies were included which examined communication patterns of physicians as well as racial disparities in EOL care delivery. Conclusions of the review show that studies repeatedly suggest that EOL discussions are lacking, likely due to the mistaken belief that patient communication is secondary when compared to the technical aspects of care. Physicians often appear to misunderstand the type of information that is valuable to patients or have difficulty assessing symptoms and needs completely (Trice \& Prigerson, 2009). In addition, the review found that physicians tend to be overly optimistic when forecasting prognoses; that what is heard by the patient during routine visits is often misunderstood or not able to be recalled; and that physicians who were more "matter of fact" were significantly more likely to care for patients who cognitively accepted their terminal condition (Trice \& Prigerson, 2009). In addition, the authors highlighted the fact that racial and ethnic disparities in cancer incidence, screening, diagnosis, treatment, and mortality have been known since the early 1990's, and there has been little change since then in terms of the presence or magnitude of these disparities 
(Trice \& Prigerson, 2009). Opportunities for "good deaths" from cancer are often missed and communication, or lack thereof, appears to be the most likely explanation for these missed opportunities. The review also noted that cost expenditures for a third of Medicare enrollees were found to be the highest in their final year of life (Trice \& Prigerson, 2009). This finding is most likely due to overly aggressive and burdensome care in the last month of life, providing evidence that the underutilization of palliative care services is ongoing and that more research is needed regarding EOL discussions (Trice \& Prigerson, 2009). Innes \& Payne (2009) conducted a review of 13 studies which examined advanced cancer patients' prognostic information preferences. The authors conducted a narrative review of the literature using a systematic approach to identify and analyze relevant studies to answer the research question. Their findings concluded that all patients wanted honesty from their professional team and the vast majority wanted some broad indication of their prognosis, but the preferences for quantitative information were more varied. The included studies used a variety of methodologies: quantitative, qualitative, and mixed methods in their designs. The consensus reached was that patients with advanced cancer want some level of prognostic information and that the benefits of awareness include: increased hope, reduced anxiety, enhanced trust, and increased satisfaction with care (Innes \& Payne, 2009). Patients identified hope as an essential element within prognostic discussions, and that discussions conducted by an expert, collaborative team enhanced feelings of hope; whereas professionals who demonstrated unease in their delivery had a detrimental effect on hope. Outcomes of the study concluded that goals of care must be addressed with patients throughout the disease trajectory and that patients must be treated individually so as to understand their personal meaning of QOL. This type 
of care requires a high level of expertise from professionals, which is likely to have additional training resource implications. The study concludes that only through a skilled approach will advanced cancer patients be provided with the appropriate level of prognostic information needed to meet their own goals and priorities in the final phase of their lives (Innes \& Payne, 2009).

\section{Oncology Nurses' Perspectives on EOL Care}

Beckstrand, Moore, Callister, \& Bond (2009) were the first to examine EOL care from the perspective of bedside oncology nurses. This major cross sectional survey invited 1,000 oncology nurses to participate in a 68 item mailed questionnaire. Respondents worked a mean of 36.1 hours per week, had been RNs for a mean of 18 years, and had worked a mean of 12.5 years in the oncology field. Respondents did not include any ambulatory nurses. In their quantitative survey, the researchers asked nurses to rank the size of obstacles and supportive behavior items in caring for patients with cancer at EOL. Eight of the first ten obstacles reviewed related to family attitudes and behaviors such as anger, denial, and over-optimism despite a patient's poor prognosis. The fifth highest reported obstacle were physicians who insisted on aggressive care until patients are actively dying (Beckstrand et al., 2009). High scoring supportive behaviors reported were: allowing families time with patients after death, having social work or palliative care available, and having family members accept that patients are dying (Beckstrand et al., 2009). Findings of the study suggested that the identification of barriers could be used to facilitate discussions and change within oncology interdisciplinary teams in order to improve EOL care. The frequency of occurrence of the obstacles and supportive behaviors were not analyzed to determine the overall magnitude of each obstacle and supportive 
behavior in the current study, but would later be reported on in Beckstrand, Collette, Callister \& Luthy's (2012) study. The four highest rated obstacles reported, all of which pertained to dealing with family members during EOL care, were also the four highest rated obstacles reported by critical care and emergency nurses in previous EOL studies conducted across multiple settings in 2005 and 2008 (Beckstrand et al., 2009).

In an effort to enhance findings from the aforementioned study, Beckstrand et al., (2012) sought to address the frequency of occurrence data to obstacle and supportive care behavior at EOL in their study. The study was conducted in an effort to determine individual item impact scores in providing EOL care. The authors asked 1,005 hospital based oncology nurses to participate in the study and had 380 respondents (37\%) to their 69 item survey. Respondents to the study were mostly experienced oncology nurses who had an average of 18 years of nursing experience. Of note, no ambulatory oncology nurses were surveyed. The highest rated obstacles by impact score reported by nurses were: dealing with anxious family members, family not accepting a poor prognosis, and being called away to help another nurse (Beckstrand et al., 2012). The top rated supportive behaviors by impact score included: allowing families time to grieve after death, providing a peaceful bedside scene for families, and allowing families unlimited access to the dying patient. The reported obstacle of physicians providing aggressive care at the EOL ranked eleventh in the 2012 study as opposed to fifth in the 2009 study (Beckstrand et al., 2012). This study, like its predecessor, cited dealing with family as vital in the provision EOL care. Results of the research demonstrate the need for more EOL education and help in forming teams of nurses, social workers, palliative care workers, and physicians to support high quality care. Communication was not a major obstacle reported in the current study, 
likely due to the fact that respondents were experienced and had developed the necessary skills needed when communicating with patients, families, and providers regarding EOL care (Beckstrand et al., 2012).

Boyd, Merkh, Rutledge, \& Randall (2011) surveyed 31 oncology nurses at a Magnet designated hospital and asked them to characterize oncology nurses' attitudes toward care at EOL and the nurses' experiences in caring for terminally ill patients, hospice discussions, and use of palliative care practices. Twenty-nine of the respondents were inpatient nurses and most reported having one or more patients with whom hospice wasn't used and probably should have been, or hospice was used but not soon enough (Boyd et al., 2011). Reasons for patients not being referred to hospice or informed they were dying included: family or patient unwillingness to accept death, physician hesitance to discuss prognosis, and nurses' hesitance to discuss prognosis. Despite these findings, the study found that oncology nurses have positive attitudes about hospice and having discussions about prognosis despite missed opportunities and hospice referrals (Boyd et al., 2011). Though limited by a small sample size, important implications for practice were identified and showed that strategies that enable nurses to have stronger voices during EOL care is needed and were, in fact, supported in practice when implemented.

In a study on nurses' attitudes toward death and caring for dying patients, Lange, Thom, \& Kline (2008) did a quantitative survey with 355 inpatient and outpatient nurses in a comprehensive cancer center in New York. The study included 141 ambulatory oncology nurses. The findings demonstrated that nurses who exhibited the most positive attitude toward caring for dying patients were those who were older and who had been employed the longest at the cancer center. Based on the results of the survey, recommendations 
included an appropriate palliative care educational component be incorporated into the new $\mathrm{RN}$ employee orientation course as well as the hospital's in-house training programs, as oncology nurses will continue to care for patients across the illness trajectory and will increasingly have to deal with death and the care of dying patients (Lange et al., 2008).

Helft, Chamness, Terry, \& Uhrich (2011) conducted a mailed pilot survey of 394 oncology nurses in order to assess their attitudes toward prognosis related communication and experiences of the quality of such communication among physicians. The study included feedback from 147 ambulatory oncology nurses. Results of the survey showed that most nurses felt they were responsible for helping patients prepare for EOL. However, almost half of the respondents felt uncertainty about what their role was in prognosis related communication and cited the uncertainty as a major barrier (Helft et al., 2011). Almost half of respondents felt that patients were unaware of a terminal prognosis, and $72 \%$ felt that physician discomfort about giving bad news was a major barrier to helping patients and families understand the prognosis. Lack of time, fear of taking away patients' hope, and "being called away from patients" were major barriers to providing EOL care. Other findings included the fact that outpatient nurses, who presumably have proximity to physicians and patients during clinical encounters, were less likely to be present for prognosis-related communication than inpatient nurses (Helft et al., 2011). In addition, despite the fact that $98 \%$ of respondents agreed or strongly agreed that nurses are responsible for initiating discussions with physicians about a patient's prognosis, $55 \%$ of them often felt pressured not to provide patients with advanced cancer prognosis-related information because they did not want to contradict what physicians had said. Implications for practice from the study were that opportunities for oncology nurses to bridge gaps in 
communication appear to exist, but that barriers such as physicians' communication need to be clarified if solutions are to be developed (Helft et al., 2011).

In an effort to explore ethical conflicts in oncology practice and the nature of healthcare contexts in which ethical conflicts can be avoided, Pavlish, Brown-Saltzman, Jakel, \& Fine (2014) surveyed 30 nurses in medical centers and community hospitals in southern California. The interviews were semi-structured and included six focus groups. The respondents did not include any ambulatory oncology nurses. The findings of the study demonstrated that nurses were frustrated by providers avoiding honest conversations about prognosis with patients and families and that the primary type of ethical conflict described by nurses occurred with seriously ill patients toward the EOL (Pavlish et al., 2014). Many of the challenges expressed by the nurses were treatment focused. The study found that delayed conversations with patients and families often reflected nurses' fears that treatments actually were harming patients. The study identifies physicians pursuing aggressive treatments at the EOL as a barrier to providing quality care to patients with advanced cancer. The study concluded that developing healthcare systems into moral communities where all members are encouraged to discuss ethical concerns in a manner that promotes trust, shared understandings, and mutual respect is necessary in oncology practice (Pavlish et al., 2014).

The review of the literature shows that oncology nurses frequently deal with patients and families at EOL and that issues such as family dynamics, poor communications, unrealistic expectations, and staffing play a major part in caring for advanced cancer patients. No studies were found that specifically addressed EOL communications from the perspective of ambulatory oncology nurses. Mcilfatrick, 
Sullivan, \& McKenna (2006) conducted a phenomenological study in Ireland in an effort to capture the experiences of oncology nurses who worked in a day hospital. The study, which included ten nurses and utilized face-to-face focused in-depth interviews, was a result of little research having been conducted thus far in more acute cancer settings. Although issues surrounding EOL were not specifically addressed, an interesting finding was that nurses viewed their role in the day hospital as being both positive and negative. The positive aspects included increased autonomy, increased skill level, and the ability to focus on the more curative aspects of cancer treatment. The negative aspects included being too task-oriented, being too technical, and being less able to provide the more caring, holistic aspects of nursing care to patients due to limitations in the physical space or because of patients' close proximity to each other during treatment, hindering privacy (Mcilfatrick, et al, 2006). The study highlighted some of the perceived obstacles of working in an ambulatory setting as well as the fact that ambulatory nurses do, indeed, face different challenges and experiences in their interactions with patients on a daily basis and may also view barriers to EOL discussions very differently than their inpatient counterparts. 


\section{Theoretical Framework}

The theoretical framework used to guide the study on barriers to end-of-life (EOL) discussions is Katherine Kolcaba's Theory of Comfort. Comfort theory is a midrange nursing theory that was developed in the early 1990's. Although relatively new, the theory has developed into a well-known one that challenges nurses to prioritize patient comfort. Within Kolcaba's theory there are three forms of comfort presented: relief, ease, and transcendence, and the three occur within the contexts of the physical, psychospiritual, environmental, and sociocultural domain (Kolcaba \& Kolcaba, 1991). Kolcaba describes comfort as holistic, and when the patient's needs of relief, ease, and transcendence are met within the four contexts, then the patient feels direct strength. Three classes of comfort needs emerge from the theory that are relevant for nursing, and each is associated with a specific sense of comfort. According to Kolcaba \& Kolcaba (1991), first there is the state sense, an enduring state of ease and peaceful contentment; second, the relief sense, which is relief from discomfort; and finally, the renewal sense, which is being strengthened and invigorated (Kolcaba \& Kolcaba, 1991).

Comfort theory has several applications to EOL care and discussions. Providers may be overly optimistic, avoid these discussions, communicate with euphemisms, and delay conversations until patients are near death (Cohen \& Nirenberg, 2011). In doing so, providers may be depriving those with advanced disease the opportunity to find closure in their lives. End-of-life discussions are difficult for providers for many reasons, including feelings of personal failure or loss, fear of upsetting patients, or fear that discussing death early in the disease course may be associated with less hope and poorer outcomes (Cohen \& Nirenberg, 2011). Although these fears are common, evidence suggests that patients suffer no psychological harm from having these discussions 
(Wright et al., 2008). Kolcaba stressed in her theory that comfort was not merely the absence of discomfort. The absence of discomfort is not a sufficient condition for the state of ease, but also requires peace of mind and cessation of intense activities (Kolcaba \& Kolcaba, 1991). Comfort theory emphasizes the whole person: the physical, the mental, and the spiritual. In order to change the current status of avoiding EOL discussions, a change in perceptions about death and the realization that death is not a failure of medicine, but is a natural part of life needs to occur.

In 1997, Kolcaba, guided by the theory of comfort, introduced a framework for the practice of hospice nursing that was based on proactive principles of comfort care. The framework served as a guide for providing individualized, holistic, and consistent comfort care to dying patients and their families (Vendlinski \& Kolcaba, 1997). Using a comfort grid, Kolcaba designed a method for nurses to assess patients' and families' needs, design interventions, and to assess the intervention's effectiveness. The concepts depicted in the grid are: relief, ease, and transcendence (types of comfort), met in the contexts of human experience (physical, psychospiritual, environmental, social) (Vendlinski \& Kolcaba, 1997). The authors also proposed that a quantitative evaluation of comfort should be taken in order to conduct empirical research in a hospice setting, either through questionnaires, or by checklists of patient/family behaviors, facial expressions, muscle tension, and statements. The framework for comfort care offers a theory-based foundation upon which to build patterned, individualized methods for the practice of comforting, which is the essence of hospice nursing (Vendlinski \& Kolcaba, 1997). 
End-of-life in cancer patients can mean many different things. Common symptoms in patients with advanced cancer include pain, fatigue, weakness, loss of appetite, and lack of energy. Moreover, symptoms increase in frequency as patients approach EOL (Greer et al., 2013). Avoiding conversations about prognosis limits discussions about goals of care and patients' wishes about how and where they want to die. Mack et al. (2012) recommended that physicians should consider moving conversations closer to diagnosis and initiating conversations while the patient is doing well, so the patient has time to plan for more difficult times in the future. According to Kolcaba \& Kolcaba, (1991), "comfort measures can bring out and amplify dispositions of character such as courage, inner strength, fortitude, and persistence", (p.1309). Treating patients and their families in an honest, holistic manner based on the principles put forth in Kolcaba's Comfort Theory will hopefully pave the way for a peaceful death, as well as provide an opportunity to achieve closure in relationships with loved ones and friends. Without these difficult conversations, patients are potentially being robbed of time spent doing things that are meaningful to them and their loves ones and, conversely, is spent in hospitals or infusion clinics receiving treatment that is, ultimately, futile. 


\section{Method}

\section{Purpose}

The purpose of the study was to explore barriers to end-of-life care discussions in cancer patients from the perspective of ambulatory oncology nurses. The purpose was also to see if the results were consistent with the existing literature, which has mainly been conducted with inpatient oncology nurses.

\section{Design}

The study design was qualitative descriptive, utilizing eight open-ended questions (Appendix A) constructed by the researcher and are based upon themes found in the literature review. A qualitative research design was chosen to better illustrate ambulatory oncology nurses attitudes about end-of-life care discussions in cancer patients. Face-toface interviews gave nurses the opportunity to speak freely about their experiences. The survey questions were piloted by two experienced oncology nurses for validity and content.

\section{Sample}

The population included ambulatory oncology nurses located at two different infusion centers, Kent Hospital, and Hematology and Oncology Associates of Rhode Island. Nurses were required to have worked in an ambulatory oncology setting for at least one year in order to participate in the study.

\section{Site}

The sites used for the study were Kent Hospital Out-Patient Infusion Unit, and Hematology and Oncology Associates of Rhode Island. Kent Hospital Infusion Unit, a Women and Infants Facility, is an 11 station facility which provides chemotherapy treatment to a variety of patients in the Kent County area and beyond. Hematology and 
Oncology Associates of Rhode Island is a 12 chair free standing infusion clinic which is privately owned and operated. The four physicians of the practice serve patients throughout RI and have privileges at Rhode Island Hospital, Kent Hospital, and Roger Williams Hospital.

\section{Procedures}

The research was reviewed by both Kent Hospital Institutional Review Board (IRB) and Rhode Island College (RIC) IRB. With approval, an informational letter (Appendix B) was sent to the nurse managers of each unit via electronic e-mail explaining the study. The e-mail was then forwarded to the nurses of each unit. Participants willing to participate were asked to notify the nurse manager, who would contact me via e-mail with dates and times for interviews. Participants were informed their participation was voluntary, and that no identifying or demographic data would be collected. Interviews were conducted in the nurse manager's offices in order to ensure privacy. Prior to the start of the interview, participants reviewed the informational letter, which also acted as a consent form, and were asked if they had any questions. The participants were aware that their answers would be recorded by a password protected audio recorder which would remain strictly in the possession of the researcher at all times. Interviews were conducted over a three day period. Each interview took between 10 and 15 minutes to conduct. The audio recordings were transcribed into a written transcript to aid in data analysis. A total of 10 nurses agreed to participate in the study. The target number of participants was between 10 and 15 . 


\section{Measurement}

The interview questions were developed by the researcher both from personal clinical experience as well as from deciphering the themes found in the existing literature regarding barriers to end-of-life care discussions in cancer patients. The literature search shed light on the fact that most of the research on this topic came from inpatient oncology nurses and not specifically from ambulatory oncology nurses. Literature themes were used as the basis for creating the eight question survey in an effort to compare if similar phenomena was experienced by the two groups of oncology nurses. Two experienced oncology nurses piloted the questions for accuracy and validity and found the questions to be sound. The piloting nurses spent about 20 minutes each reviewing the questions as well as approximating length of time for each interview.

\section{Data Analysis and Storage}

The literature review did not yield a survey regarding nurses personal perceptions about barriers to end-of-life care discussions in cancer patients and so therefore, the researcher created the questionnaire and piloted the questions with two experienced oncology nurses. Answers from each interview were made into a transcript which the researcher read through and highlighted and sorted by similar themes. The data was then used to compare themes found in the literature review in an effort to determine if answers were similar for both inpatient and ambulatory oncology nurses. Data was stored on a password protected audio recorder which was kept locked in a drawer at the researcher's home. Data collected will be saved for a minimum of six months after project completion.

Results will be reported. 


\section{Results}

A total of ten female nurses participated in the study, six from Kent Hospital and four from Hematology and Oncology Associates of RI. The number of years spent in ambulatory oncology ranged from three and a half to twenty-five, with an average of sixteen years. At Kent, physicians are not on site while patients are being treated and nurses do not have access to office notes. At Hematology and Oncology Associates, the physicians' offices are on site and they are often in the building while patients are being treated. The infusion nurses have access to all of the physicians' office notes.

When asked about their experiences caring for patients at or near end-of-life who are receiving aggressive chemotherapy, seven of the nurses reported feeling conflicted about this, two reported not feeling conflicted, and one reported being conflicted only sometimes. Of those who felt conflicted, statements included things such as: patients' time being better served at home with loved ones instead of getting sick from chemotherapy, and patients not having to spend the end of their life sitting in chairs receiving medications that aren't going to help them. One nurse stated, "I wonder if they realize what the outcome is going to be. I realize it's not my place to judge but what's the point if it gives them only one or two more weeks". Another nurse said that it is difficult to treat when you know that there is nothing else that can be done for patients, but that as long as there is quality of life, she could consider it to be worthwhile as long as the patient has made an informed decision. One nurse with 25 years of experience stated that she is not always conflicted because she believes most patients know when enough is enough, and if they are choosing to continue treatment near the end, then she will give them what they want without feeling conflicted. Of the nurses who reported not 
feeling conflicted about treating with aggressive chemotherapy, one nurse stated that she felt it was her job to give the patient what they wanted without judgment, another nurse stated that as long as the side effects weren't debilitating to the patient then she did not personally have a problem with it.

When asked if they had a problem providing prognostic information to patients about their disease, nine of the study participants reported that they had no problem with this, and the nurse with the least number of years of experience (3.5) stated that she is not comfortable. Answers included such things as patients being more comfortable speaking with nurses rather than doctors; wanting to answer questions honestly but encouraging patients to live one day at a time; answering in general terms based on stage of disease but also encouraging patients to speak to their physician if they are looking for more specific answers. One nurse stated that if the patient is asking then that means they want to know, and that she was comfortable giving information depending on how the question was phrased. She stated, "Am I going to die tomorrow is an easy one. Mostly likely the answer is no. How long do I have to live is a difficult one because I don't know and neither does the doctor really know". Two of the nurses from Kent stated that they don't avoid it but that they would feel better knowing exactly what the physician has told the patient already and that they are not privy to that information in the infusion unit. One nurse with 25 years of experience stated that patients know exactly what is going on whether they have been told prognostic information or not. The least experienced ambulatory nurse stated she is not comfortable with this topic and that she would be fearful of contradicting what the physician has told the patient. 
When asked if they felt that patients who were near end-of-life were aware of their prognosis, answers were varied. Five felt that patients were not aware, one felt that they were, and four stated that they were unsure or that the patient is not always aware. Among those who felt that they were not aware, reasons given were that physicians don't like to give up and that patients don't always hear what is said. Others felt patients may be in denial. One nurse stated, 'Medical oncologists don't want to tell patients that they are near the end of life". Yet another stated "You know, sometimes I just don't think that we spell it out enough". Of the nurses who stated they were unsure of this answer, the reason given was they could not be sure if patients were aware because they don't have access to what has been told to them by the physicians. She stated, "I wish I knew what the physicians say or are telling them because we want to be on the same page". The one nurse who felt that patients are aware of their prognosis said that it probably takes physicians longer than it should to deliver that information to patients but that she believed patients know when they are at the end even when no one has spoken it. Another nurse felt that often times it is the family who does not want the patient to be told their prognosis and that she feels as though she is being asked to lie to the patient at times. As an example she stated that she had been asked by a family member on one occasion to please not say the word "chemotherapy" to the patient, but to simply call it "treatment".

Participants were asked what they felt were the biggest barriers to physicians not having end-of-life care discussions with their patients with cancer. Many of the answers were the same, particularly ones surrounding physicians not wanting to take away hope or admitting that there is nothing more that can be done for a patient. Also, physicians' 
comfort level was mentioned as a barrier. This type of response was given by eight of the ten nurses. Another reason given by seven of the nurses was physicians not having enough time to spend on these conversations, which are typically difficult and lengthy. Reasons cited included physicians having to see a high volume of patients in the course of a day and knowing that they have a waiting room full of sick patients waiting for their time. Four of the nurses said that physicians may feel as though they have failed the patient, or that they may have difficulty delivering bad news. One nurse stated that patients put so much faith and trust in their physician that it must be very difficult telling someone who has put their life in your hands that you have nothing left to offer them. Other reasons cited as barriers by two of the nurses were family members who don't want to accept that their loved one is at the end, and who make it very difficult for bad news to be given to patients. The nurse with the least number of years of experience felt that physicians do not focus on end-of-life at all. She stated, "I don't even think they are thinking about it".

The next question asked if the participants were ever a part of end-of-life discussions between patients and physicians and the answer given from each nurse was that they were not. The second part of the question asked if they felt that they would like to be a part of those discussions and the answers varied. Three of the nurses stated they would possibly like to be part of the conversation depending upon the relationship they had with the patient. They stated that they felt that it would benefit the patient if everyone were on the same page. One nurse stated that it would be helpful to learn how the news is given and a few stated that they would like to be present in order to give a nurse's perspective about end-of-life. Another nurse stated that she felt it would be 
beneficial to be part of the discussions in order to increase awareness and involvement in end-of-life care, which she believed was just as important as treating the patient for the cancer. This nurse stated that she believed end-of-life discussions should take place in the initial meetings between physicians and patients in the same way that chemotherapy, surgery, and radiation are discussed. Another felt that if the nurse was present for those meetings, then a door for discussion would be opened and everyone would be on the same page. Two nurses stated that they would prefer just to have access to the scans and notes from the physicians so that they knew how to answer specific questions that may be asked of them. They did not, however, want to be involved in the actual conversations.

The next question asked of the participants was if they felt that they should be part of end-of-life discussions between patients and physicians. Answers were varied, with seven of the nurses stating that they did feel they should be part of the discussions, and three preferring to being privy to the results of such discussions. Of the ones who stated that they should be part of end-of-life discussions, reasons cited included being able to obtain services earlier on for patients; being there as an extra support for patients and relaying pertinent information to the physician with regards to the patient's treatment; giving a more personal feel to the meetings between patients and physicians, as nurses often know more personal information about their patients; and also to feel that they are a part of the patient's team, which encompasses all aspects of a patient's care. Two nurses stated that they felt they are missing a piece of the puzzle by not being involved in end-of-life discussions and that it does not give them the whole picture. All of the nurses stated they did not believe that they would care for their patients any 
differently if they were taking part in end-of-life care discussions, but did believe it would lead to having more honest conversations with them near the end of life.

The final question asked pertained to patients wanting to have a discussion about transitioning to end-of-life care and what kind of resources or support is available to the nurses. All of the nurses stated that they would be willing to have such discussions with their patients and that they would personally reach out to someone to assist in the patient's transition. Three of the nurses stated they would have no problem calling and speaking to the physician directly. Most of them said they would reach out to a social worker on the patient's behalf. Kent has a social worker on site two days a week, and Hem/Onc Associates has a social worker who makes visits to the office when consulted. Three of the Kent nurses mentioned getting the nurse navigator or the nurse practitioner involved if they were asked to discuss transitioning to end-of-life. Others stated they would give literature about local hospice and palliative care agencies to patients if asked. One nurse stated that she would reach out to Kent's Palliative Care Department if she needed help obtaining a referral for a patient. A few of the nurses noted that if a patient requested to speak privately about transitioning to end-of-life, they would find a private space in which to have a suitable conversation with them. All of the nurses stated that they would, in fact, assist patients in their effort to transition to end-of-life care. 


\section{Summary and Conclusions}

Cancer is among the leading causes of death worldwide, is the second leading cause of death in the U.S., and is the leading cause of death in those between the ages of 45 and 64 years (Kochanek et al., 2011). In the U.S., we are falling short of the IOM's goal that those with palliative needs deserve access to appropriate expert-level care and those who meet the criteria of a six month or less prognosis should be offered hospice referral (IOM, 2014). Barriers to implementation to early EOL care in oncology have been reviewed extensively in the literature. The SUPPORT trial was one of the first studies to examine EOL in hospitalized patients and identified EOL care obstacles leading to a decreased QOL for terminally ill patients (Connors, et al., 1995). There is an abundance of literature regarding oncology nurses and their perceptions of EOL care. Beckstrand, et al. (2009), were the first to examine EOL care from the perspective of bedside oncology nurses. In their survey, the researchers asked nurses to rank the size of obstacles and supportive behavior items in caring for patients with cancer at EOL (Beckstrand et al., 2009). A review of the literature, however, showed that no studies have been conducted regarding barriers to EOL care discussions solely from the perspective of ambulatory oncology nurses. Ambulatory oncology nurses are in a unique position to add to the discussion surrounding barriers to EOL care due to the abundant amount of time spent with patients while they are receiving treatment. For this reason, a qualitative study was conducted on the perceived barriers to EOL care discussions in cancer patients from the perspective of ambulatory oncology nurses.

The study was guided by Katherine Kolcaba's Comfort Theory, which challenges nurses to prioritize patient comfort. Comfort theory emphasizes the whole person: the physical, the mental, and the spiritual. In 1997, Kolcaba, guided by the theory of 
comfort, introduced a framework for the practice of hospice nursing that was based on the proactive principles of comfort care. Guided by this framework, the researcher conducted an eight question interview style survey with ten ambulatory oncology nurses. The questions were based on those found in the existing literature and were piloted by two experienced oncology nurses. The nurses surveyed worked at two different sites, Kent Hospital Out-Patient Infusion Unit, and Hematology and Oncology Associates of RI, a private physician owned practice. The nurses had a wide range of experiences, from three to twenty-five years. Limitations of the study were all of the nurses surveyed were white women, and there was not a great deal of ethnic diversity among the patient population at the two facilities.

The results of the study concluded that both inpatient and outpatient oncology nurses face similar challenges in dealing with EOL discussions with cancer patients. Similarities included feeling conflicted about the use of aggressive chemotherapy near EOL, not wanting to contradict any information that the physician has told a patient, as well as the belief that physicians are not having EOL discussions with patients until there is nothing left to offer them. Both groups of oncology nurses cite similar barriers to physicians not having timely discussions with their patients about transitioning to EOL care. These barriers included: physicians own lack of comfort; not wanting their patients to lose hope or lose trust in them; lack of time, due to either high patient volume or due to the length of time such conversations require; as well as physicians not wanting to disappoint patients because they are not able to cure them.

The study also showed that overall, both inpatient and outpatient nurses have positive feelings about being involved in and providing EOL care, as well as being 
involved in conversations with physicians regarding transitions to EOL care for patients. Ambulatory nurses in the study all reported not being involved in discussions between patients and physicians, even those who work with the physicians on site. Inpatient oncology nurses have more opportunities to speak with physicians and may have the benefit of being a part of a team when patients are admitted, thereby allowing them to be directly involved in EOL care discussions. Despite these opportunities, they report being frustrated by physicians lack of having timely EOL discussions, as well as by providing aggressive treatments to patients who are at or near the end-of-life. A major difference noted between the two groups in the study was that inpatient nurses reported dealing with family members as a major obstacle in the provision of EOL care. This, no doubt, reflects the logistical differences between the two groups. Inpatient nurses face the issue of patients being much sicker when they are admitted and perhaps being hospitalized for several days. Family members are often present at the hospital, and this adds a tremendous burden to inpatient nurses, especially if the patient is not doing well. Inpatient nurses have the added burden of caring not only for their patients, but also for anxious and stressed family members as well. Unlike their counterparts, ambulatory oncology nurses typically see patients when they are functional and perhaps not as ill. More than likely, ambulatory patients bring only one or two family members to the clinic due to the limited amount of space in the ambulatory setting. Family members were not cited as a major barrier for the provision of EOL care by ambulatory oncology nurses. Ambulatory oncology nurses reported feeling as though they are not part of the patient's team, or feeling like there is a missing piece of a puzzle by their lack of involvement in EOL care discussions. Some reported being unsure if patients are even 
aware of their prognosis. They reported a willingness to bring a nurse's view to the discussion, as well as a desire for more open and honest communication with patients. Many reported they felt a need to be informed so that they could be better advocates for palliative and hospice care. As a result of the disconnect among them, their patients, and physicians, ambulatory oncology nurses often feel frustrated about the gap that exists in their ability to care for cancer patients who are at or near end-of-life. All of the nurses surveyed stated they would not hesitate to provide assistance or to find resources for patients requesting information about EOL care. They reported a willingness to do this on their own, and also by seeking advice from nurse navigators, nurse practitioners, as well as social workers. All of the nurses who were surveyed, with the exception of the one with the least amount of experience (3 years) also reported being comfortable giving prognostic information to their patients themselves, but feel they are unable to do so because such information is not being shared with them by physicians. 


\section{Recommendations and Implications for Advanced Nursing Practice}

Based on the study, there are many recommendations and implications for advanced nursing practice regarding EOL care discussions in cancer patients. Advanced practice nurses, especially oncology nurses, may act as liaisons between physicians, nurses, and patients in order to assure that there is adequate communication among all parties. Oncology patients deserve to have all members of their team on the same page, so that they are best able to make informed decisions regarding their care and in planning for their future. The literature review identified that patients want honest information about their prognosis and that the provision of such provides patients with more hope, improved survival, reduced depression, and lower resource use when prognostic discussions were had (Smith, et al, 2012). Advanced practice nurses may, in fact, be the team member who facilitates EOL care transitions for patients and the one who provides the necessary resources for patients and families. Additionally, the APRN may be the one who, upon seeing a decline in a patient's condition, begins the discussion with the physician about the transition to EOL care. They may also serve as the intermediary between physicians and oncology nurses in relaying necessary information about a patient's condition.

The advanced practice nurse has the ability to fill many gaps in oncology nursing, but perhaps none as vital as the one in which they serve as a catalyst in the patient's transition to EOL care. The literature shows that, in spite of the necessity and the benefit of earlier and effective EOL care, the United States continues to fall short in the timely use of palliative and hospice care services, and that Medicare spending is at it's highest in a patient's final month of life (Trice \& Prigerson, 20009). Advanced practice nurses in 
oncology are in a position to help achieve the IOM's goal of providing appropriate and expert-level care to the terminally ill, both by taking a more active role in the way EOL care discussions are handled as well as in fostering excellent communication among all members of the patient's team. This may be accomplished both by being a judicious advocate for the use of EOL care in the terminally ill, as well as by encouraging physicians to have earlier EOL care discussions with their terminally ill patients. 


\section{References}

American Cancer Society. (2014). A guide to palliative or supportive care. Retrieved fromwww.cancer.org/treatment/treatmentsandsideeffects/palliativecare/supportive -care

American Cancer Society. (2016a). Cancer facts \& figures 2016. Retrieved from http://www.cancer.org/acs/groups/content/@ research/documents/document/acspc047079.pdf

American Cancer Society. (2016b). What is hospice care? Retrieved from http://www.cancer.org/treatment/findingandpayingfortreatment/choosingyou rtreatmentteam/hospicecare/hospice-care-hospice-care

Beckstrand, R., Moore, J., Callister, L., \& Bond, E. (2009). Oncology nurses’ perceptions of obstacles and supportive behaviors at the end of life. Oncology Nursing Forum, $36(4), 446-453$.

Beckstrand, R., Collette, J., Callister, L., \& Luthy, K. (2012). Oncology nurses’ obstacles and supportive behaviors in end-of-life care: Providing vital family care.

Oncology Nursing Forum, 39(5), 398-406.

Bonebrake, D., Culver, C., Call, K., \& Ward-Smith, P. (2010). Clinically differentiating palliative care and hospice. Clinical Journal of Oncology Nursing, 14(3), 273275.

Boyd, D., Merkh, K., Rutledge, D., \& Randall, V. (2011). Nurses’ perceptions and experiences with end-of-life communication and care. Oncology Nursing Forum, $38(3), 229-239$.

Cohen, A., \& Nirenberg, A. (2011). Current practices in advance care planning. Clinical Journal of Oncology Nursing, 15(5), 547-553. 
Connors, A.F., Dawson, N.V., Desbiens, N., Fulkerson, W.J., Goldman, L., Knaus, W., \& Ransohoff, D. (1995). A controlled trial to improve care for seriously ill hospitalized patients. The study to understand prognoses and preferences for outcomes and risks of treatments (SUPPORT). JAMA, 274(20), 1591-1598.

Davis, S., Lind, B., \& Sorenson, C. (2013). A comparison of burnout among oncology nurses working in adult and pediatric inpatient and outpatient settings. Oncology Nursing Forum, 40(4), 303-311.

Greer, J., Jackson, V., Meier, D., \& Temel, J. (2013). Early integration of palliative care services with standard oncology care for patients with advanced cancer. $C A$ : $A$ Cancer Journal for Clinicians, 63, 349-363.

Helft, P., Chamness, A., Terry, C., \& Uhlrich, M. (2011). Oncology nurses' attitudes toward prognosis-related communication: A pilot mailed survey of Oncology Nursing Society members. Oncology Nursing Forum, 38(4), 468-474.

Innes, S., \& Payne, S. (2009). Advanced cancer patients' prognostic information preferences: A review. Palliative Medicine, 23, 29-39.

Institute of Medicine. (2014). Dying in America. IOM committee's proposed core components of quality end-of-life care. Retrieved from http://www.nationalacademies.org/hmd/ /media/Files/Report\%20Files/2014/EOL /Table\%20-\%20Core\%20Components\%20of\%20Quality\%20Care.pdf

Keating, N., Landrum, M.B., Rogers, S., Baum, S., Virnig, B., Huskamp, H., Earle, C., \& Kahn, K. (2010). Physician factors associated with discussions about end-of-life care. Cancer, 116(4), 998-1006. 
Kochanek, K.D., Jiaquin, X., Murphy, S.1., Minino, A.M., \& Hsian-Ching, K. (2011). Deaths: Final Data for 2009. Centers for Disease Control and Prevention, National Vital Statistics Reports, 60(3), tables 9,10,11. Retrieved from http://www.cdc.gov/nchs/data/nvrs/nvrs60 03.pdf

Kolcaba, K., \& Kolcaba, R. (1991). An analysis of the concept of comfort. Journal of Advanced Nursing, 16, 1301-1310.

Lange, M., Thom, B., \& Kline, N. (2008). Assessing nurses' attitudes toward death and caring for dying patients in a comprehensive cancer center. Oncology Nursing Forum, 35(6), 955-959.

Laryionava, K., Heubner, P., Hiddeman, W., \& Winkler, E. (2014). Framework for timing of the discussion about forgoing cancer-specific treatment based on a qualitative study with oncologists. Support Care Cancer, 23(3), 715-721.

Mack, J., Cronin, A., Keating, N., Taback, N., Huskamp, H., \& Malin, J. (2012). Associations between end-of-life discussion characteristics and care received near death: A prospective cohort study. Journal of Clinical Oncology, 30(35), 43874395.

Mcilfatrick, S., Sullivan, K., \& McKenna, H. (2006). Nursing the clinic vs. nursing the patient: nurses' experience of a day hospital chemotherapy service. Journal of Clinical Nursing, 15(9), 1170-1178.

Moir, C., Roberts, R., Martz, K., Perry, J., \& Tivis, L. (2015). Communicating with patients about palliative and end-of-life care: Comfort and educational needs of nurses. International Journal of Palliative Nursing, 21(3), 109-112. 
National Cancer Institute. (2016). Cancer statistics. Retrieved from http://www.cancer.gov/about-cancer/understanding/statistics

National Comprehensive Cancer Network. (2011). Practice Guidelines in Oncology Palliative Care. Retrieved from https://www.nccn.org/professionals/physician_gls/pdf/palliative.pdf

Pavlis, C., Brown-Saltzman, K., Jakel, \& Fine, A. (2014). The nature of ethical conflicts and the meaning of moral community in oncology practice. Oncology Nursing Forum, 41(2), 130-140-

Sharma, N., Sharma, A., Wojtowycz, M., Wang, D., \& Gajra, A. (2015). Utilization of palliative care and acute care services in older adults with advanced cancer. Journal of Geriatric Oncology, 7(2016), 39-46.

Smith, T., Temin, S., Alesi, E., Abernathy, A., Balboni, T., Basch, E., ... Von Roenn, J. (2012). American Society of Clinical Oncology provisional clinical opinion: The integration of palliative care into standard oncology care. Journal of Clinical Oncology, 30 (8), 880-887.

Trice, E., \& Prigerson, H. (2009). Communication in end-stage cancer: Review of the literature and future research. Journal of Health Community, 14(0 1), 95-108.

Vendlinski, S. \& Kolcaba, K. (1997). Comfort care: A framework for hospice nursing. The American Journal of Hospice \& Palliative Care, November/December, 271276.

Von Gunten, C., Lutz, S., \& Ferris, F. (2011). Why oncologists should refer patients earlier to hospice care. Oncology, 25(13), Retrieved from http://www.cancernetwork.com 
Zhou, G., Stolftzfus, J., Houldin, A., Parks,S., \& Swan, B.A. (2010). Knowledge, attitudes, and practice behaviors of oncology advanced practice nurses regarding advanced care planning for patients with cancer. Oncology Nursing Forum, 37(6), 400-410. 
Appendix A

Survey Questions

Perceived Barriers to End-of-Life Discussions in Cancer Patients from the Perspective of Ambulatory Oncology Nurses

1. Can you tell me how many years you have been working as an ambulatory oncology nurse?

2. Can you tell me about your experiences caring for cancer patients who are near the end-of-life and who are receiving aggressive chemotherapy? Do you ever feel conflicted about treating those patients?

3. Do you feel comfortable having conversations with patients if they ask you prognostic information about their illness, or would you rather not have those types of discussions with them?

4. Do you feel that, for the most part, cancer patients who are near end-oflife have been made aware of their prognosis by their provider? If so, what signs have you seen that suggest that they are aware?

5. What, if any, do you see as the biggest barriers to providers having discussions with their patients about transitioning to end-of-life care?

6. Are you ever given the opportunity to be part of end-of-life discussions between your patients and their providers? If no, would you like to be?

7. Do you feel that you should be part of end-of-life care discussions between patients and physicians? Do you think that doing so would change the way you care for patients in any way?

8. What would you do if a patient wanted to have a discussion about transitioning to end-of-life care? Do you have any support systems in place for staff or patients to have those types of conversations? 


\section{Appendix B}

\section{Informational Letter}

\section{Perceived Barriers to End-of-Life Care Discussions from the Perspective of}

\section{Ambulatory Oncology Nurses}

My name is Susan Hobin and I am a master's student at Rhode Island College in the Adult Acute Care Nurse Practitioner program. You are being asked to participate in a research study about barriers to end-of-life care discussions in cancer patients. You are being asked because you have worked in an ambulatory oncology setting for at least one year. Please read this letter and ask any questions you may have before choosing to participate in the study. My contact information can be found at the end of this letter.

\section{Why this Study is Being Done (Purpose)}

I am conducting this study to explore ambulatory oncology nurses perceptions about barriers to end-of-life care discussions in cancer patients.

\section{What You Will Have to Do (Procedures)}

If you choose to be in this study, I will ask you to meet with me at a time suitable to you and your nurse manager. You will partake in a face-to-face interview which will be audio recorded. Questions asked will be open-ended and conversational and will address endof-life issues in cancer patients from your own perspective. The interview should take approximately 20 minutes and will be conducted in a space agreed upon by you and your nurse manager.

\section{Risks or Discomforts}

There are no right or wrong answers to the survey questions. They are meant to be conversational and informative and are merely seeking your opinion. Should you, however, experience any emotional distress related to the interview questions, you may contact Todd Ellison, LICSW, at 401-237-4494.

\section{Benefits}

There are no direct benefits to you participating in the study. There is minimum risk to you if you choose to participate.

\section{Deciding to be in the Study}

Being part of the study is completely voluntary. You can change your mind if you so choose and there will be no penalty to you.

\section{How Your Information will be Protected}

Because this is a research study, results will be recorded and later transcribed by a third party, and then summarized by the researcher in a major paper. There will be no identifying data collected and your name will not be used anywhere. The transcript will later be used in a research paper in order to discuss the themes of the findings and to see how these findings compare to those of other oncology nurses. Upon completion of the 
survey, audio recordings will be disposed of in a shredder once transcript is made. Audio recordings will not be shared with anyone besides the researcher. The transcript will be stored on a password protected computer for a period of six months upon project completion. Results of this paper will be shared with fellow nursing students and nursing faculty in the Spring of 2017 at the Rhode Island College Master's Symposium.

Who to Contact : Susan Hobin 401-451-0485 - $\quad$ Linda Dame - ldame@ @ic.edu 